\title{
Relationship between nutritional biomarkers and occlusal status in gastric cancer patients using the Eichner Index: A cross sectional study
}

Atsushi Abe ( $\square$ atsushi.a@ekisai.or.jp )

Nagoya Ekisaikai Hospital https://orcid.org/0000-0001-8215-2769

Yu Ito

Aichi-Gakuin University

Hiroki Hayashi

Nagoya Ekisaikai Hospital

Takanori Ishihama

Nagoya Ekisaikai Hospital

Eri Umemura

Nagoya Saisyukan Hospital

Akari Tsunoda

Nagoya Ekisaikai Hospital

Research article

Keywords: tooth loss, occlusal status, prognostic nutritional index, postoperative infection

Posted Date: April 24th, 2020

DOl: https://doi.org/10.21203/rs.3.rs-23517/v1

License: (c) (1) This work is licensed under a Creative Commons Attribution 4.0 International License.

Read Full License 


\section{Abstract \\ Background}

Systemic inflammatory responses and nutritional status are useful prognostic factors in patients with gastric cancer. Because oral hypofunction is a cause of undernutrition, we cross-sectionally investigated whether some nutritional biomarkers were affected by occlusal supporting zone status.

\section{Methods}

The study included 114 patients ( 85 men and 29 women) diagnosed with gastric cancer at Nagoya Ekisaikai Hospital between September 2014 and March 2019. Gastric cancer stage, body mass index (BMI), albumin levels, total lymphocyte counts, cholesterol levels, and C-reactive protein levels were evaluated in preoperative blood tests. Four kinds of nutritional biomarkers were evaluated; they are the Glasgow prognostic score (GPS), neutrophil-lymphocyte ratio (NLR), prognostic nutrition index (PNI), and controlling nutritional status (CONUT). Oral conditions were assessed by determining the number of remaining teeth, occlusal supporting status based on the Eichner classification. Patients were assigned into three groups per their occlusal status, and mean values were compared using the Kruskal-Wallis test.

\section{Results}

The mean age and BMI were $72.2 \pm 8.5(50-89)$ years and $22.0 \pm 3.6(14.8-33.4)$, respectively. There were here were $42,39,23$, and 10 stages I, II, III, and IV patients, respectively. The mean number of remaining teeth was $18.1 \pm 9.5$ teeth. According to the Eichner classification, there were 45, 42, and 27 patients in groups $A, B$, and $C$, respectively. There were significant differences in the GPS and NLR values, and CONUT frequencies between groups $\mathrm{A}$ and $\mathrm{C}(P=0.033, P=0.00097, P=0.04$, respectively) (MannWhitney U test). PNI values were lower in Group $C$ with poor occlusal support zones than in Group A with stable occlusal support zones.

\section{Conclusions}

Reductions in occlusal supporting zones were associated with undernutrition. Notably, patients in Eichner Class $\mathrm{C}$ with few occlusal supporting zones had poor GPS, PNI, and CONUT values, and were found to be undernourished. Thus, patients with markedly reduced occlusal contacts are often undernourished and have advanced systemic inflammation, which may affect prognosis. Patients in the Eichner Class $\mathrm{C}$ group need interventions to improve nutritional status such as dietary counseling due to potential nutritional deficits experienced by this group of patients.

\section{Background}


Gastric cancer is one of the most lethal forms of cancer worldwide, with an estimated 951,600 new cases and 723,100 deaths in 2012 [1, 2]. Despite early detection, improvements in surgical procedures, and availability of advanced chemotherapeutic options, long-term survival is still unsatisfactory due to the associated local recurrence or distant metastasis. The immune and nutritional status of patients have been reported to be associated with postoperative outcomes in malignant tumors [3-6]. The nutritional status and systemic inflammatory responses in patients with cancer are considered to be useful prognostic factors [7-9].

The nutritional status of patients with cancer is often evaluated by physical examination and blood biochemical findings such as albumin [10]. Patients with gastric cancer who are immunocompromised due to preoperative undernutrition or systemic inflammatory responses are often have delayed wound healing, develop complications, and have increased mortality rates [11-13]. Malnutrition often causes changes that can lead to a poor prognosis $[14,15]$. Therefore, it is important to develop a precise biomaker to objectively evaluate patients with malnutrition. Various biomarkers that reflect the inflammatory state, nutritional status, and immunocompetence of patients have been reported as valuable prognostic factors [16-20]. One method of evaluation is to measure plasma components such as C-reactive proteins (CRP) or serum albumin (ALB) levels, another involves measuring blood cells such as neutrophil or lymphocytes levels, and a third method involves the combination of these two. Use of a nutritional evaluation index has advantages and when used with other nutritional indexes, it can help to improve the diagnoses of patients. Due to the limitations of using a single index, we chose appropriate nutritional indexes depending on the disease stage and the type of therapy patients received [16]. Preoperative undernutrition is due to multiple biological, physiological, and pathological factors, one of which is a decreased oral function. Oral dysfunction, which causes undernutrition, includes impaired masticatory ability due to tooth loss, dry oral mucosa, and infection. Such conditions affect food choices and result in a reduced quality of life (QOL), development of severe complications, and increased mortality rates [16-20]. If cancer progresses, the nutritional status of patients gradually worsens. However, patients with a decreased oral cavity function with no disease progression tend to tilt towards being malnourished. Hence, a decrease in the oral cavity function results in a poor preoperative nutritional status during gastric cancer surgery, with the possibility of an increase in the occurrence of postoperative complications. Although there have been studies that investigated how a decrease in the number of teeth and an impaired masticatory function affect nutritional status, studies that determine the association between nutritional status before gastrointestinal cancer surgery and oral conditions of patients are few. Specifically, no study has focused on the relationship between biomarkers and occlusal zone support status exhaustively. In a previous study, we reported that the Prognostic Nutrition Index (PNI) differed significantly between group A (those with occlusal zone support) and group $\mathrm{C}$ (those with no occlusal zone support based on the Eichner classification [21-23]. There are two kinds of biomarkers for evaluating systemic inflammation and nutritional status. Currently, it is uncertain whether the intraoral state of the patient affects either blood cells or plasma components and to determine these effects would require a novel evaluation approach. We showed in our previous work that the assessment of nutritional status using the PNI and ALB levels is important in preoperative dental evaluation. 
In the present cross-sectional study, we investigated whether some biomarkers (Glasgow prognostic score [GPS], neutrophil-lymphocyte ratio [NLR], controlling nutritional status [CONUT], and PNI), preoperative nutritional markers in patients with gastric cancer, would differ according to occlusal support zone status. A change in food selection is often observed with a decline in masticatory efficiency due to tooth loss and malocclusion [24]. People with chewing difficulties eventually consume less nutritious foods such as vegetables, fruits, meats and grains [24-26]. As a result, blood levels of vitamins and protein are reduced; however, the nutritional evaluation index is thought to be more accurately reflected by evaluating plasma protein levels $[27,28]$. This is because a change in diet is considered to have a bigger impact on plasma proteins than on other hematopoietic mechanisms in the short term. For this study, using the GPS, CONUT, and PNI, we evaluated the plasma components that are affected by occlusion support zone status. Additionally, we used the NLR to assess how other blood components are affected by occlusion support zone status. From this assessment, we determined how the occlusion support zone status is associated with various biomarkers in the blood.

\section{Methods}

\section{Subjects}

This cross-sectional study enrolled 145 patients who were pathologically diagnosed with primary gastric cancer, and underwent a dental examination before surgery at the Nagoya Ekisaikai Hospital between September 2014 and March 2019. Of these patients, 31 who had recurrent cancer, metabolic diseases (e.g., diabetes mellitus), or incomplete data were excluded. The present study, 114 patients (85 men and 29 women) were included.

\section{Assessment of gastric cancer and general conditions}

Gastric cancer was diagnosed by consensus among skilled physicians. While assessing gastric cancer, the onset site and cancer stage was determined based on the results of endoscopy, computed tomography, and magnetic resonance imaging performed at the department of gastrointestinal surgery. The body mass index (BMI) of each patient was also calculated. Blood samples were collected on admission, during the presurgical examination (within one month before surgery). From these blood samples, ALB levels, total lymphocyte counts, and CRP levels were measured. Based on these preoperative blood test results, the severity of inflammation was assessed.

\section{Evaluation of nutritional biomarker}

We evaluated four kinds of nutritional biomarkers. The biomarkers evaluated were GPS [29, 30], NLR [31, 32], CONUT [33, 34], and PNI $[17,18]$ as described in Table 1. The GPS was calculated using preoperative CRP and ALB values. Patients with raised concentrations of CRP (over $10 \mathrm{mg} / \mathrm{L}$ ) and hypoalbuminemia $(A L B<35 \mathrm{~g} / \mathrm{L})$ were given a score of 2 . Those with raised concentrations of CRP without hypoalbuminemia were given a score of 1 . Patients who did not have raised concentrations of CRP were given a score of 0 regardless of their ALB status. Neutrophil-lymphocyte ratios were also calculated. A 
neutrophil-lymphocyte ratio of $\geq 5$ was given a score of 1 ; if it was $<5$, the score was 0 . PNI was calculated on the basis of serum ALB levels and peripheral blood lymphocyte count using the following formula. 
Table 1

Inflammation-based prognostic scores

\begin{tabular}{|c|c|c|}
\hline Factor & Group & Overall \\
\hline$n$ & & 114 \\
\hline \multirow[t]{2}{*}{ Denture (0:yes, 1: no; \%) } & 0 & $45(39.5)$ \\
\hline & 1 & $69(60.5)$ \\
\hline \multirow[t]{3}{*}{ Eichner.1.A.2.B.3.C (\%) } & 1 & $45(39.5)$ \\
\hline & 2 & $42(36.8)$ \\
\hline & 3 & $27(23.7)$ \\
\hline \multirow[t]{2}{*}{ Sex (0: male, 1: female; \%) } & 0 & $85(74.6)$ \\
\hline & 1 & $29(25.4)$ \\
\hline \multirow[t]{4}{*}{ Stage (\%) } & 1 & $41(36.0)$ \\
\hline & 2 & $39(34.2)$ \\
\hline & 3 & $25(21.9)$ \\
\hline & 4 & $9(7.9)$ \\
\hline Age & & $71.83(10.00)$ \\
\hline Alb & & $3.71(0.57)$ \\
\hline BMI & & 21.99 (3.57) \\
\hline CRP & & $0.67(1.54)$ \\
\hline Lymph & & $26.09(8.22)$ \\
\hline Neutrophil & & $65.07(8.91)$ \\
\hline NLR & & $3.14(2.78)$ \\
\hline $\mathrm{PNI}$ & & $40.42(6.25)$ \\
\hline Remaining teeth & & $18.10(9.54)$ \\
\hline Total lymph & & 1643.47 (851.77) \\
\hline WBC & & $6.94(8.60)$ \\
\hline Age & & $74.00[28.00,89.00]$ \\
\hline Alb & & $3.80[1.80,4.70]$ \\
\hline
\end{tabular}

Alb: albumin; BMI: body mass index; CRP: C-reactive protein; NLR: neutrophil-lymphocyte ratio; PNI: prognostic nutrition index; WBC: white blood cells 


\begin{tabular}{|c|c|c|}
\hline Factor & Group & Overall \\
\hline BMI & & $21.85[14.80,33.40]$ \\
\hline CRP & & $0.17[0.02,10.53]$ \\
\hline Lymph & & $25.90[3.90,45.80]$ \\
\hline Neutrophil & & $63.95[45.00,92.50]$ \\
\hline NLR & & $2.46[0.98,23.72]$ \\
\hline PNI & & $40.82[19.13,52.33]$ \\
\hline Remaining teeth & & $22.00[0.00,31.00]$ \\
\hline Total lymph & & $1520.75[230.10,7872.00]$ \\
\hline WBC & & $5.80[3.00,96.00]$ \\
\hline
\end{tabular}

$\mathrm{PNI}=[10 \times$ serum albumin level $(\mathrm{g} / \mathrm{dL})]+\left[0.005 \times\right.$ total peripheral lymphocyte count $\left(\right.$ per $\left.\left.\mathrm{mm}^{3}\right)\right]$

The serum albumin concentration, total peripheral lymphocyte count and total cholesterol concentration were used to obtain the CONUT score. The scores were added up and patients were categorized into the following groups based on the cumulative score: normal ( $0-1)$ mild disorder (2-4), moderate disability $(5-8)$, and severe disability $(>8)$.

\section{Assessment of oral conditions}

Oral conditions were assessed by two dentists who were blinded to patients' nutritional status in the present study. In the oral examination, the remaining teeth excluding residual dental roots were counted, and the occlusal support zone status was assessed according to the Eichner classification [7]. The Eichner classification is a method used to evaluate occlusion support zone status based on the remaining teeth. The zone is defined as a site supporting occlusion. In a healthy dentition composed of opposing tooth pairs in the right and left premolar and molar regions, occlusion is supported by 4 occlusal supporting zones. The Eichner index categorizes the occlusal status into three main groups $(A, B$, and $\mathrm{C}$ ). The number of remaining teeth and Eichner classification are so objective that they can be regarded as very reliable.

In patients using dentures, their dentures were examined by a dentist. The occlusal supporting zones were assessed for contact between opposite teeth pairs.

\section{Statistical analysis}

The number of cases in the area during the study period determined the sample size. We also examined whether the values of the four biomarkers differed among the 3 Eichner Classes A, B, and C. We 
considered whether there was a difference in GPS and CONUT frequencies between the three Eichiner groups. Categorical data (GPS and CONUT) were analyzed using $\chi^{2}$ tests, except when expected cells were found to be less than 5 , in which case, Fisher's exact test was employed. The Shapiro-Wilk test was used to assess the distribution of preoperative NLR and PNI values, and the normality was confirmed at a $5 \%$ significance level. Because NLR and PNI were not normally distributed $(P=0.000003493$ and 0.02542), the three groups were compared using the Kruskal-Wallis test, which is a non-parametric test. In addition, the Steel-Dwass multiple comparison tests were performed to determine which of the three groups showed differences in NLR and PNI. All statistical analyses were performed with EZR (Saitama Medical Center, Jichi Medical University, Saitama Japan), which is a graphical user interface for $R$ (The R Foundation for Statistical Computing, Vienna, Austria). More precisely, it is a modified version of $\mathrm{R}$ commander designed to add statistical functions frequently used in biostatistics [24]. Based on the effect size observed in our study, the estimated sample size was 44 patients per group and a total of 88 patients. Sample size estimates were based on the following: standard deviation within groups 5.0, alevel, 0.5 and power of $80 \%$

\section{Ethics}

This cross-sectional study was approved by the Nagoya Ekisaikai Hospital Ethics Committee (approval number 2017-040). The study was conducted in accordance with the Strengthening the Reporting of Observational Studies in Epidemiology (STROBE) Statement guidelines for reporting observational studies.

\section{Results}

\section{Patient characteristics}

None of the markers of systemic and oral conditions were normally distributed. Patients were classified into three groups according to the occlusal support zone status based on the Eichner classification to compare each marker. Table 1 shows patient characteristics. The mean age of the patients was $72.2 \pm$ 8.5 years (range, $50-89$ years). The mean BMI was $22.0 \pm 3.5$ (range, 14.8-33.4). There were 42, 39, 23, and 10 stages I, II, III, and IV patients, respectively. According to the Eichner classification, there were 45, 42 , and 27 patients in groups $A, B$, and C, respectively. No significant differences were observed in sex, age, gastric cancer stage, BMI, or CRP levels among the three Eichner Classes A, B, and C. Concerning denture usage, 45 of the 143 patients used dentures, and 69 did not. Among these patients using dentures, the number of those without occlusal supports was 4, 22, and 19 in groups A, B, and C, respectively. (Table 2 ). 
Table 2

Patient characteristics

\begin{tabular}{|c|c|c|c|c|c|}
\hline & & Eichner & & & \\
\hline Factor & Group & $A$ & B & C & $P$ value \\
\hline $\mathrm{n}$ & & 45 & 42 & 27 & \\
\hline Denture (\%) & Yes & $4(8.9)$ & $22(52.4)$ & $19(70.4)$ & $<0.001$ \\
\hline & No & $41(91.1)$ & $20(47.6)$ & $8(29.6)$ & \\
\hline Sex (\%) & Male & $32(71.1)$ & $33(78.6)$ & $20(74.1)$ & 0.725 \\
\hline & Female & $13(28.9)$ & $9(21.4)$ & $7(25.9)$ & \\
\hline Stage (\%) & 1 & $16(35.6)$ & $13(31.0)$ & $12(44.4)$ & 0.734 \\
\hline & 2 & $16(35.6)$ & $13(31.0)$ & $10(37.0)$ & \\
\hline & 3 & $10(22.2)$ & $12(28.6)$ & $3(11.1)$ & \\
\hline & 4 & $3(6.7)$ & $4(9.5)$ & $2(7.4)$ & \\
\hline Age & & $68.51(10.81)$ & 74.21 (8.86) & $73.67(9.00)$ & 0.015 \\
\hline Alb & & $3.91(0.45)$ & $3.68(0.51)$ & $3.43(0.71)$ & 0.002 \\
\hline BMI & & $21.98(3.23)$ & 22.69 (3.53) & $20.94(4.04)$ & 0.137 \\
\hline CRP & & $0.47(1.09)$ & $0.49(0.78)$ & $1.26(2.61)$ & 0.07 \\
\hline Lymph & & 27.44 (7.99) & $26.04(7.84)$ & $23.92(8.98)$ & 0.214 \\
\hline Neutrophil & & $63.89(8.70)$ & $64.80(7.88)$ & $67.46(10.53)$ & 0.251 \\
\hline NLR & & $2.66(1.26)$ & $2.97(1.92)$ & $4.22(4.84)$ & 0.061 \\
\hline $\mathrm{PNI}$ & & $42.49(4.87)$ & $40.34(5.81)$ & $37.11(7.59)$ & 0.001 \\
\hline Total lymph & & $1687.57(592.20)$ & $1760.62(1152.40)$ & $1387.72(609.94)$ & 0.188 \\
\hline WBC & & $6.24(1.65)$ & $8.34(14.00)$ & $5.93(1.74)$ & 0.412 \\
\hline
\end{tabular}

\section{Oral conditions}

The mean number of remaining teeth was $18.1 \pm 9.5$ teeth (range, $0-31$ teeth). Even among patients with the same number of remaining teeth, if the number of occlusal supporting zones differs, their nutritional status may be differently affected. Thus, differences in nutritional biomarkers were analyzed according to the Eichner classification. The nutritional biomarkers are shown in the Table 2 according to Eichner 
Classes A, B, and C. The CRP levels were $0.5 \pm 1.1,0.5 \pm 0.7$, and $1.7 \pm 3.3$ in groups $A, B$, and $C$, respectively $(P=0.01)$, indicating significant differences among the three groups. The ALB levels were 3.9 $\pm 0.45,3.68 \pm 0.5$, and $3.43 \pm 0.7$ in groups $\mathrm{A}, \mathrm{B}$, and $\mathrm{C}$, respectively $(P=0.002)$, indicating significant differences among the three groups. (Table 2 )

The GPS is a common score used to assess levels of inflammation. The score assessments revealed frequencies of 32 GPS level 0, 4 GPS level 1, 5 GPS level 2, and 4 GPS level 3 in group A, 21 GPS level 0, 9 GPS level 1, 6 GPS level 2, and 6 GPS level 3 in group B, and 11 GPS level 0, 2 GPS level 1, 3 GPS level 2, and 11 GPS level 3 in group $C(P=0.0179)$, indicating significant differences among the three groups. In addition, multiple comparisons revealed no difference in GPS levels between groups $A$ and $B$, and between groups $\mathrm{B}$ and $\mathrm{C}(P=0.615, P=0.23)$, but significant differences were observed between groups $\mathrm{A}$ and $\mathrm{C}(P=0.033)$ (Mann-Whitney U test) (Fig. 1).

The NLR (nutrient rating system using blood cells) values were $2.7 \pm 1.3,3.0 \pm 1.9$, and $4.2 \pm 4.8$ in groups $\mathrm{A}, \mathrm{B}$, and $\mathrm{C}$, respectively $(P=0.217)$, indicating no significant difference among the three groups. (Fig. 2$)$.

The PNI is calculated based on the serum albumin concentration and peripheral blood lymphocyte count. The values for patients in this study were $42.6 \pm 4.9,40.3 \pm 5.8$, and $37.1 \pm 7.6$ in groups $A, B$, and $C$, respectively $(P=0.0048)$, indicating significant differences among the three groups. In addition, multiple comparisons revealed no difference in either ALB levels or PNI between groups $A$ and $B$, and between groups $\mathrm{B}$ and $\mathrm{C}(P=0.29, P=0.06)$, but significant differences were observed between groups $\mathrm{A}$ and $\mathrm{C}$ ( $P$ $=0.00097)$ (Mann-Whitney U test). These results revealed that PNI values were lower in Group $C$ with poor occlusal support than in Group A with stable occlusal support (Fig. 3).

The CONUT score is calculated according to serum albumin concentration, total peripheral lymphocyte count and total cholesterol concentration. The score frequencies for this assessment were 25 CONUT level 1, 15 CONUT level 2, 4 CONUT level 3, and 1 CONUT level 4 in group A, 15 CONUT level 1, 16 CONUT level 2, 10 CONUT level 3, and 1 CONUT level 4 in group B, and 6 CONUT level 1, 12 CONUT level 2, 5 CONUT level 3 , and 4 CONUT level 4 in group $C(P=0.03)$, indicating significant differences among the three groups. In addition, multiple comparisons revealed no difference in GPS levels between groups $\mathrm{A}$ and $\mathrm{B}$, and between groups $\mathrm{B}$ and $\mathrm{C}(P=0.39, P=0.6)$, but significant differences were observed between groups $\mathrm{A}$ and $\mathrm{C}(P=0.04)$ (Mann-Whitney $\mathrm{U}$ test) (Fig. 4).

Thus, the present study revealed that the GPS, PNI, and CONUT were affected by the numbers of remaining teeth and occlusal supporting zones.

\section{Discussion}

The oral cavity is an important organ for nutrient intake, and nutritional status is affected by impaired oral function due to hypofunction of the temporomandibular joints, masticatory muscles, missing teeth, and impaired swallowing function [35-37]. In the present study, the examination of occlusal support zone status and nutritional biomarkers revealed significant differences in GPS and CONUT levels and PNI values. The Eichner C group without occlusal support had higher GPS levels than did Group A, and the 
CONUT levels revealed moderate or severe malnutrition; PNI values were low in this group. However, there was no significant difference in NLR between the three groups. Furthermore, nutritional evaluations using the protein levels of the blood revealed significant differences in the oral cavity function failure among the three groups; whereas, a significant difference was not found in nutritional evaluation using the blood cells. Therefore, the change in diet due to oral cavity function disorder reduced plasma proteins such as albumin and cholesterol, and was thought to result in undernutrition and poor immunocompetence. The results suggested that impaired oral function due to oral diseases and tooth loss reduces dietary intake and is associated with undernutrition. The correlation between the number of residual teeth and nutritional status has been reported in many studies, and the results of the present study are comparable with those of such studies. Meanwhile, the bite force varies depending on periodontal disease and stability of the remaining teeth, and it affects the food choice and nutritional status. Thus, even among patients with the same number of remaining teeth, their conditions may differ depending on the sites and arrangement of the remaining teeth.

Plasma proteins decrease due to undernutrition arising from poor caloric intake. The decrease in plasma proteins reduces immunocompetence, and the presence of inflammation worsens the situation. Although the Eichner classification is based on the assessment of the occlusal support zone status at the time of clinical examination, it may help make a diagnosis of oral frailty [37] after a certain period because the simultaneous loss of multiple teeth rarely occurs. Thus, this classification was used in the present study. A comparison among the 3 Eichner groups revealed significant differences in GPS, CONUT, and PNI among the groups, and multiple comparisons revealed a significant difference between groups $\mathrm{A}$ and $\mathrm{C}$. It is rare to lose many teeth in a short period of time; the patients in group $\mathrm{C}$ might were likely undernourished before they were diagnosed with gastric cancer.

In patients with poor preoperative nutritional status, the relative risks of postoperative complications and in-hospital death are considered to be increased by 2-4 folds. Cell-mediated immunity is most affected by undernutrition. Undernourished people exhibit atrophy of the thymus, reduced peripheral T cell counts, reduced CD4/CD8 ratios (CD4 dominant), and atrophy of lymph nodes, spleen, and gut-associated lymphoid tissue (GALT), which is the immune tissue of the intestinal tract. Atrophy of GALT reduces the immunity of the intestinal mucosa and causes gastrointestinal infection. In addition, severe malnutrition reduces immunoglobulin levels. On the other hand, neither neutrophil nor peripheral B lymphocyte count decreases; but the phagocytic capacity, bactericidal capacity, and complement production are reduced [38-41].

Furthermore, systemic inflammatory responses are reported to reflect immune responses and are associated with prognosis. Systemic inflammatory responses are useful for predicting prognosis via a mechanism different from that of conventional tumor markers [41, 42]. Many tumor markers are tumor antigens and substances secreted by tumors that are measured directly and assessed. On the other hand, systemic inflammatory responses are an indirect measure of cancer progression. This is considered to be due to the following mechanism: host immunity is activated by tumor recognition, and proinflammatory 
cytokines are released in blood as intracellular mediators. Consequently, acute inflammatory proteins increase; thus, host immune responses are enhanced as tumors progress [43].

Biomarkers for assessing systemic inflammatory responses and the nutritional status include the Glasgow prognostic score [29, 30], neutrophil/lymphocyte ratio [31, 32], lymphocyte/monocyte ratio [44, 45], and CRP/ALB ratio [46]. These markers are calculated based on serum protein levels (e.g., CRP and ALB), cell counts (e.g., neutrophil, lymphocyte, and monocyte), and ratios of these values. Each nutrient rating system has its intrinsic characteristics. There are limitations to the use of a single index; thus, we chose appropriate nutritional indexes and evaluated them based on the disease stage and the therapeutic option employed.

The GPS was the index reported in non-small lung cell cancer by Macmillan, and the GPS combines CRP and ALB and evaluates blood protein moieties primarily. The GPS has been reported in various cancer studies to be a prognostic marker. The thing that comes to have a poor prognosis is reported in the systemic inflammatory reaction sthenia case like GPS Category 2, 3. The poor case shows systemic inflammatory such as the GPS Category 2 and 3 and undernutrition. The CRP level of patients with cancer reflects IL- 6 levels in the blood, and a persistent increase in IL-6 levels suggests inflammation of the carcinoma tissue [47]. Whereas, the progression of periodontal disease and the presence of dental caries cause odontogenic infectious diseases and an increase in CRP.

However, between the three Eichner groups, a significant difference was not found in CRP levels. The effect on GPS had little impact on teeth and chronic odontogenic infectious diseases in this examination, while for effect on GPS category, ALB was thought to be more important. Systemic inflammatory responses are assessed as changes in biochemical data that result from activation of the immune system by the presence of tumors and secretion of IL-6 and other proinflammatory cytokines [47-49].

NLR is a nutrient rating system using blood cells (neutrophils and lymphocytes). Lymphocytes have been used as one of the nutritional evaluation indexes for a long time [50]. We are used for an index of the immunocompetence that the lymphocytes are related to tumor immunity and act for a tumor restrainingly. The lymphocytes are related to tumor immunity and act for a tumor restrainingly [51, 52]. Whereas neutrophils increase due to inflammation and induce the production of chemokines and cytokine, the produced chemokines and cytokines enhance the growth, invasion, and neovascularization of tumors. Therefore, tumor growth is closely associated with inflammation [53, 54]. NLR, which evaluates CRP and ALB ratios, is reported as an oncological prognostic marker similar to GPS. A significant difference was not found in this examination between the three Eichner groups. NLR is considered to mirror a balance between the innate and adaptive immune mechanisms [54]. The NLR can reflect the initial innate immune mechanisms (involving cells such as neutrophils and macrophages that provide a nonspecific response), which triggers the adaptive immune mechanisms (T-cell/B-cell mediated, and partly PLT stimulated) that result in periodontal destruction. In cases of high NLR values, periodontitis becomes more severe [52]. It is reported that NLR is useful for clinical evaluations of periodontitis. A mouth cleaning state is good in Eichiner A where many residual teeth are present in, and 
inflammatory reaction is poor. Additionally, there is no effect as we raise CRP because Eichner $\mathrm{C}$ has few residual teeth causing odontogenic infectious diseases. The difference was not found in such situation. Whereas there are many patients that a lot of residual teeth have good mouth cleaning state in Eichner $\mathrm{A}$. Also, Eichner $\mathrm{C}$ has few residual teeth and does not reach it before we greatly change CRP even if periodontal disease is severe because there are few teeth causing inflammation. A difference was not found from such situation. PNI and CONUT are indexes that are dependent on the evaluation of lymphocyte counts, ALB, and cholesterol. It has been reported that it has a poor prognosis in low level of $\mathrm{PNI}$ and the extensive malnutrition category of CONUT. The lymphocytes have antitumor immunity eligibility. The lymphocytes commit a tumor restrainingly and have antitumor immunocompetence [53]. Cholesterol half-life in the blood is eight days, shorter than 21 days for ALB, and reflects malnutrition subtly [24-26, 55]. Significant differences were found between the three groups and PNI and CONUT proved to the more reflective of undernutrition in group $C$ than in group A. Wakai et al. [55] reported that as the number of teeth decreases, people consume fewer vegetables but their total calorie intake increases with an increase in the consumption of carbohydrate such as rice, and other confectionaries. If the occlusion status is poor, carbohydrates and glucide play key dietary roles. Simultaneously, there is a reduced intake of neutral fat or proteins. As a result, these indexes are thought to worsen.

The present study has several limitations. Nutritional status is affected by many factors. In addition to oral findings such as prosthodontic treatment with dentures and conditions of the remaining teeth, other factors including socioeconomic strata, income levels, and acquired behavioral patterns are also very important [52]. It was impossible to control all of these potential variables in the present study.

Furthermore, to determine whether dentures functioned well, factors that can influence outcomes such as the levels of technical skills of dentists, designs of dentures, the difficulty of cases, skills of dental technicians who fabricate dentures, perfection levels of dentures, and the actual use of dentures, should be assessed. However, there are no standardized methods to assess these factors, and only a few studies have reported the evaluation of these factors. These factors should be assessed in future studies. Because the present study is a cross-sectional study, no causal relationship could be determined. Thus, we cannot state that the number of teeth or occlusal status directly affects nutritional status.

An early transition to oral intake is essential in the treatment of gastric cancer. The reasons for this are not only that oral intake is the best enteral nutrition, but also that eating through the mouth is an important activity, which is associated with human dignity and affects QOL. In addition to the problems of undernutrition, prolonged lack of oral intake leads to a disuse hypofunction of the eating and swallowing muscles. Thus, a delayed start of oral intake due to complications, such as postoperative ruptured suture, should be avoided, and efforts should be made to precisely diagnose and improve preoperative undernutrition. Given the importance of the function of the remaining teeth for nutrient intake and the unduly significant effects of impaired masticatory function on nutritional status, dentists and dental hygienists in a nutrition support team play an important role in maintaining and improving oral function, which is important for nutrient intake. For example, the oral cavity should be kept clean, and the periodontal condition should be maintained. In addition, patients with occlusal disharmony may be undernourished, as an underlying condition, even if they are satisfied with denture use. Thus, occlusal 
disharmony is suggested to be a possible factor for the consideration of preoperative nutritional supplementation. Based on this study, there are two conclusions that can be drawn regarding malnutrition and inflammation in patients with gastric cancer patients with oral health conditions: 1) There are direct effects of occlusal abnormalities on dietary intake, and 2) There is indirect action of systemic inflammatory conditions such as odontogenic infectious diseases on acute protein synthesis.

Further studies on oral health and its role in malnutrition and inflammation are warranted. A long-term prospective study and intervention study are necessary to completely evaluate the association among systemic inflammation in oral health, malnutrition, and gastric cancer.

\section{Conclusions}

The association between the nutritional biomarkers and dental conditions of patients with gastric cancer with different occlusal statuses was examined. Decreases in the occlusal supporting zones were correlated with undernutrition. Patients with fewer occlusal supporting zones had lower ALB levels and $\mathrm{PNI}$, and GPS and CONUT levels were found to be reduced in undernourished patients. The Eichner $\mathrm{C}$ group was inferior to other groups in GPS, PNI, CONUT.The occlusion status affects humoral ingredients such as albumin. This study emphasizes an important role of oral health in improving malnutrition in gastric cancer. Furthermore, it shows that it predicts the decrease of a nutrient in the gastric cancer and the caloric intake that the oral cavity appearing by the loss of teeth is in bad health. Furthermore, oral functional decline due to the tooth missing shows that we predict a decrease in nutrition of patients with gastric cancer and systemic inflammatory reaction.

\section{Abbreviations}

PNI

Prognostic nutrition index

GPS

Glasgow Prognostic Score

CRP

C-reactive protein

ALB

albumin

CONUT

Controlling Nutritional Status

NLR

Neutrophil-lymphocyte ratio

BMI

body mass index

\section{Declarations}


Ethics approval and consent to participate: This cross-sectional study was approved by the Nagoya Ekisaikai Hospital Ethics Committee (approval number 2017-040), and written informed consent was obtained from all the patients before participating in the study.

Consent for publication: Not applicable

Availability of data and materials: All data generated or analyzed during this study are included in this published article.

Competing interests: The authors declare that they have no competing interests.

Funding: The present research did not receive any specific grant from funding agencies in the public, commercial, or not-for-profit sectors.

Authors' contributions: AA conceived the study, carried out design and coordination and wrote the manuscript and gave the final approval of the version to be submitted. EU and YI critically revised the manuscript for important intellectual content. AT, HH and TI collected the clinical data and drafted the article. All authors read and approved the final manuscript.

Acknowledgments: We would like to thank Editage Science Communications for English language editing and publication support.

\section{References}

1. Torre LA, Bray F, Siegel RL, Ferlay J, Lortet-Tieulent J, Jemal A. Global cancer statistics, 2012. CA Cancer J Clin. 2015;65:87-108.

2. Shen L, Shan YS, Hu HM, Price TJ, Sirohi B, Yeh KH, et al. Management of gastric cancer in Asia: resource-stratified guidelines. Lancet Oncol. 2013;14:e535e47.

3. Yoo HM, Lee HH, Shim JH, Jeon HM, Park CH, Song KY. Negative impact of leakage on survival of patients undergoing curative resection for advanced gastric cancer. J Surg Oncol. 2011;104:734-40.

4. Sierzega M, Kolodziejczyk P, Kulig J, Polish Gastric Cancer Study Group. Impact of anastomotic leakage on long-term survival after total gastrectomy for carcinoma of the stomach. Br J Surg. 2010;97:1035-42.

5. Schwegler I, von Holzen A, Gutzwiller JP, Schlumpf R, Muhlebach S, Stanga Z. Nutritional risk is a clinical predictor of postoperative mortality and morbidity in surgery for colorectal cancer. Br J Surg. 2010;97:92-7.

6. Ray-Coquard I, Cropet C, Van Glabbeke M, Sebban C, Le Cesne A, Judson I, et al. Lymphopenia as a prognostic factor for overall survival in advanced carcinomas, sar- comas, and lymphomas. Cancer Res. 2009;69:5383-91.

7. McMillan DC. An inflammation-based prognostic score and its role in the nutrition-based management of patients with cancer. Proc Nutr Soc. 2008;67:257-62. 
8. McMillan DC. The systemic inflammation-based Glasgow Prognostic Score: a decade of experience in patients with cancer. Cancer Treat Rev. 2013;39:534-40.

9. Diakos $\mathrm{Cl}$, Charles KA, McMillan DC, Clarke SJ. Cancer-related inflammation and treatment effectiveness. Lancet Oncol. 2014;15:e493-503.

10. Alshadwi A, Nadershah M, Carlson ER, Young LS, Burke PA, Daley BJ. Nutritional considerations for head and neck cancer patients: A review of the literature. J Oral Maxillofac Surg. 2013;71(11):185360. doi:10.1016/j.joms.2013.04.028.

11. Soini H, Muurinen S, Routasalo P. Oral and nutritional status: Is the MNA a useful tool for dental clinics. J Nutr Health Aging. 2006;10:495-9. discussion 500-1.

12. De Marchi RJ, Hugo FN, Hilgert JB, Padilha DM. Association between oral health status and nutritional status in south Brazilian independent-living older people. Nutrition. 2008;24:546-53.

13. Yoshida M, Suzuki R, Kikutani T. Nutrition and oral status in elderly people. Jpn Dent Sci Rev. 2014;50:9-14.

14. Müller-Richter U, Betz C, Hartmann S, Brands RC. Nutrition management for head and neck cancer patients improves clinical outcome and survival. Nutr Res. 2017;48:1-8.

doi:10.1016/j.nutres.2017.08.007.

15. Z LC,H. J. Y, et al. Survival and prognostic analysis of preoperative inflammatory markers in patients undergoing surgical resection for laryngeal squamous cell carcinoma. BMC Cancer. 2018;18(1):816. doi:http://dx.doi.org/10.1186/s12885-018-4730-x.

16. Kinoshita A, Onoda H, Imai N, Iwaku A, Oishi M, Fushiya N, et al. Comparison of the prognostic value of inflammation-based prognostic scores in patients with hepatocellular carcinoma. $\mathrm{Br} \mathrm{J}$ Cancer. 2012;107:988-93. doi:10.1038/bjc.2012.354.

17. Yang $Y$, Gao P, Song Y, Sun J, Chen X, Zhao J, et al. The prognostic nutritional index is a predictive indicator of prognosis and postoperative complications in gastric cancer: A meta-analysis. Eur $\mathrm{J}$ Surg Oncol. 2016;42:1176-82. doi:10.1016/j.ejso.2016.05.029.

18. Namikawa T, Ishida N, Tsuda S, Fujisawa K, Munekage E, Iwabu J, et al. Prognostic significance of serum alkaline phosphatase and lactate dehydrogenase levels in patients with unresectable advanced gastric cancer. Gastric Cancer. 2019;22:684-91.

19. Namikawa T, Kawanishi Y, Fujisawa K, Munekage E, Iwabu J, Munekage M, et al. Serum carbohydrate antigen 125 is a significant prognostic marker in patients with unresectable advanced or recurrent gastric cancer. Surg Today. 2018;48:388-94.

20. McMillan DC, Forrest LM, O'Gorman P, Angerson WJ, McArdle CS. Performance status of male and female advanced cancer patients is independently predicted by mid-upper arm circumference measurement. Nutr Cancer. 2002;42:191-3.

21. Ship JA, Duffy V, Jones JA, Langrnore S. Geriatric oral health and its impact on eating. J Am Geriatr Soc. 1996;44:456-64.

22. Fukai K, Takiguchi T, Ando Y, Aoyama H, Miyakawa Y, Ito G, et al. Mortality rates of communityresiding adults with and without dentures. Geriatr Gerontol Int. 2008;8:152-9. 
23. Abe A, Kurita K, Hayashi H, Ishihama T, Ueda A. Correlation between prognostic nutritional index and occlusal status in gastric cancer. Oral Dis. 2020;26:1-8. doi:10.1111/odi.13242.

24. Tsakos G, Herrick K, Sheiham A, Watt RG. Edentulism and fruit and vegetable intake in low-income adults. J Dent Res. 2010;89:462-7.

25. Ritchie CS, Joshipura K, Hung HC, Douglass CW. Nutrition as a mediator in the relation between oral and systemic disease: associations between specific measures of adult oral health and nutrition outcomes. Crit Rev Oral Biol Med. 2002;13:291-300.

26. Nowjack-Raymer RE, Sheiham A. Association of edentulism and diet and nutrition in US adults. J Dent Res. 2003;82:123-6.

27. Okamoto N, Amano N, Nakamura T, Yanagi M. Relationship between tooth loss, low masticatory ability, and nutritional indices in the elderly: A cross-sectional study. BMC Oral Health. 2019;19(1):110. doi:10.1186/s12903-019-0778-5.

28. Gaewkhiew P, Sabbah W, Bernabé E. Does tooth loss affect dietary intake and nutritional status? A systematic review of longitudinal studies. J Dent. 2017;67(June 2017):1-8. doi:10.1016/j.jdent.2017.10.012.

29. Forrest LM, McMillan DC, McArdle CS, Angerson WJ, Dunlop DJ. Comparison of an inflammationbased prognostic score (GPS) with performance status (ECOG) in patients receiving platinum-based chemotherapy for inoperable non-small cell lung cancer. Br J Cancer. 2004;90:1704-6.

30. Zhang CX, Wang SY, Chen SQ, Yang SL, Wan L, Xiong B. Association between pretreatment Glasgow prognostic score and gastric cancer survival and clinicopathological features: a meta-analysis. Onco Targets Ther. 2016;9:3883-91.

31. Shimada H, Takiguchi N, Kainuma O, Soda H, Ikeda A, Cho A, et al. High preoperative neutrophillymphocyte ratio predicts poor survival in patients with gastric cancer. Gastric Cancer. 2010;13:1706.

32. Proctor MJ, McMillan DC, Morrison DS, Fletcher CD, Horgan PG, Clarke SJ. A derived neutrophil to lymphocyte ratio predicts survival in patients with cancer. Br J Cancer. 2012;107:695-9.

33. Ignacio de Ulíbarri J, González-Madroño A, de Villar NG, González P, González B, Mancha A, et al. CONUT: a tool for controlling nutritional status. First validation in a hospital population. Nutr Hosp. 2005;20:38-45.

34. González-Madroño A, Mancha A, Rodríguez FJ, Culebras J, de Ulibarri Jl. Conforming the validity of the CONUT system for early detection and monitoring of clinical undernutrition: comparison with two logistic regression models developed using SGA as the gold standard. Nutr Hosp. 2012;27:564-71.

35. Takata Y, Ansai T, Awano S, Hamasaki T, Yoshitake Y, Kimura Y, et al. Relationship of physical fitness to chewing in an 80-year-old population. Oral Dis. 2004;10:44-9.

36. Yoshida M, Morikawa H, Yoshikawa M, Tsuga K, Akagawa Y. Eight-year mortality associated with dental occlusion and denture use in community- dwelling elderly persons. Gerodontology. 2005;22:234-7. 
37. Ikebe K, Matsuda K, Kagawa R, Enoki K, Yoshida M, Maeda Y, et al. Association of masticatory performance with age, gender, number of teeth, occlusal force and salivary flow in Japanese older adults: is ageing a risk factor for masticatory dysfunction? Arch Oral Biol. 2011;56:991-6.

38. Diakos $\mathrm{Cl}$, Charles KA, McMillan DC, Clarke SJ. Cancer related inflammation and treatment effectiveness. Lancet Oncol. 2014;15:e493-503.

39. Roxburgh CS, McMillan DC. Cancer and systemic inflammation: treat the tumour and treat the host. Br J Cancer. 2014;110:1409-12.

40. Douglas E, McMillan DC. Towards a simple objective framework for the investigation and treatment of cancer cachexia: the Glasgow Prognostic Score. Cancer Treat Rev. 2014;40:685-91.

41. Deguchi H, Tabuchi Y, Saitoh Y. Histopathological and immunohistochemical studies on the elevation mechanism of cancer associated antigens, CEA and CA19-9, in patients with colorectal cancer. Nihon Geka Gakkai Zasshi. 1988;89:671-83.

42. Miller CL. Immunological assays as measurements of nutritional status: a review. JPEN J Parenter Enteral Nutr. 1978;2:554-66.

43. McMillan DC. The systemic inflammation-based Glasgow Prognostic Score: a decade of experience in patients with cancer. Cancer Treat Rev. 2013;39:534-40.

44. Nishijima TF, Muss HB, Shachar SS, Tamura K, Takamatsu Y. Prognostic value of lymphocyte-tomonocyte ratio in patients with solid tumors: A systematic review and meta-analysis. Cancer Treat Rev. 2015;41:971-8.

45. Huang Y, Feng JF. Low preoperative lymphocyte to monocyte ratio predicts poor cancer-specific survival in patients with esophageal squamous cell carcinoma. Onco Targets Ther. 2015;8:137-45.

46. Ishizuka M, Nagata H, Takagi K, Iwasaki Y, Shibuya N, Kubota K. Clinical significance of the c-reactive protein to albumin ratio for survival after surgery for colorectal cancer. Ann Surg Oncol. 2016;23:900-7.

47. Grivennikov SI, Greten FR, Karin M. Immunity, inflammation, and cancer. Cell. 2010;140:883-99.

48. Huber C, Bobek N, Kuball J, Thaler S, Hoffarth S, Huber C, et al. Inhibitors of apoptosis confer resistance to tumour suppression by adoptively transplanted cytotoxic T-lymphocytes in vitro and in vivo. Cell Death Differ. 2005;12:317-25.

49. Tazzyman S, Lewis CE, Murdoch C. Neutrophils: key mediators of tumour angiogenesis. Int J Exp Pathol. 2009;90:222-31.

50. Gregory AD, Houghton AM. Tumor-associated neutrophils: new targets for cancer therapy. Cancer Res. 2011;71:2411-6.

51. Azab B, Camacho-Rivera M, Taioli E. Average values and racial differences of neutrophil lymphocyte ratio among a nationally representative sample of united states subjects. PLoS One. 2014;9:e112361.

52. Song $X$, Chen $D$, Yuan $M$, Wang $H$, Wang Z. Total lymphocyte count, neutrophil-lymphocyte ratio, and platelet-lymphocyte ratio as prognostic factors in advanced non-small cell lung cancer with 
chemoradiotherapy. Cancer Manag Res. 2018;10:6677-83.ã⿰冈日.

53. McMillan DC. Systemic inflammation, nutritional status and survival in patients with cancer. Curr Opin Clin Nutr Metab Care. 2009;12:223-6.

54. Josse JM, Cleghorn MC, Ramji KM, Jiang H, Elnahas A, Jackson TD, et al. The neutrophil-tolymphocyte ratio predicts major perioperative complications in patients undergoing colorectal surgery. Colorectal Dis. 2016;18:236-42.

55. Wakai K, Naito M, Naito T, Kojima M, Nakagaki H, Umemura O, et al. Tooth loss and in- takes of nutrients and foods: a nationwide survey of Japanese dentists. Community Dent Oral Epidemiol. 2010;38:43-9.

\section{Figures}




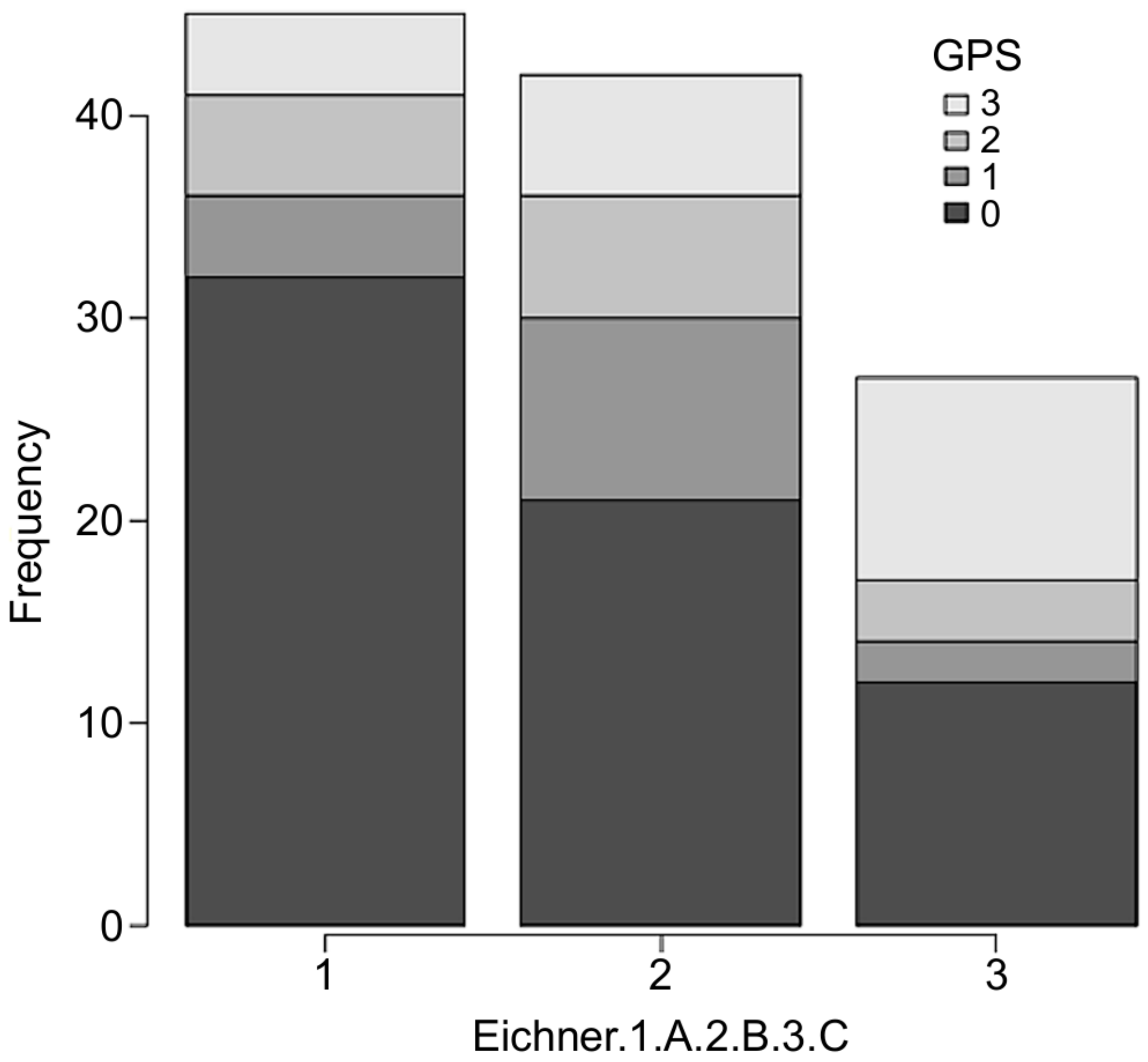

Figure 1

Comparison of GPS levels among the three groups based on Eichner index. GPS: Glagow prognostic score 


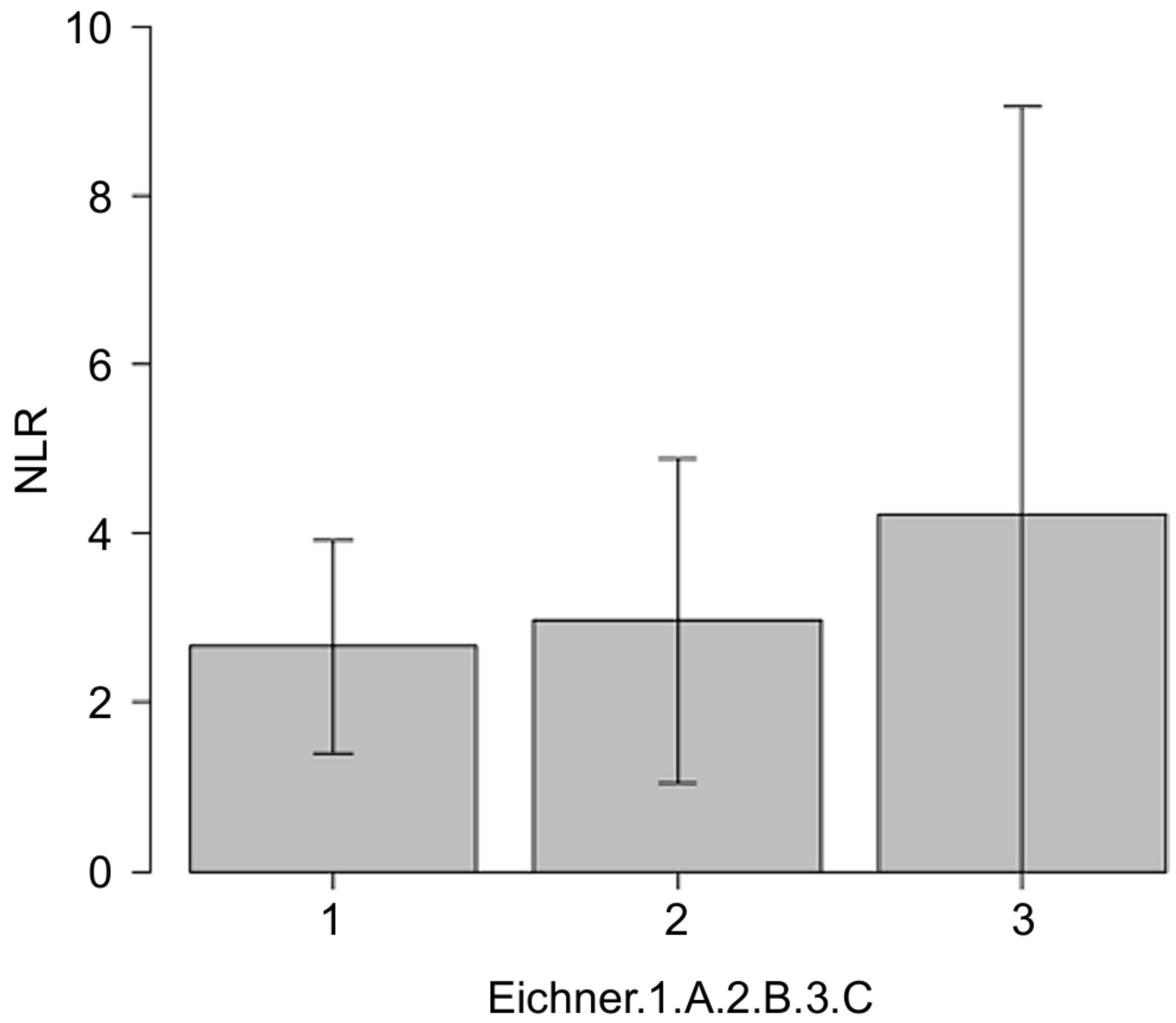

Figure 2

Comparison of PNI levels among the three groups based on Eichner index. PNI: Prognositic nutrition index 


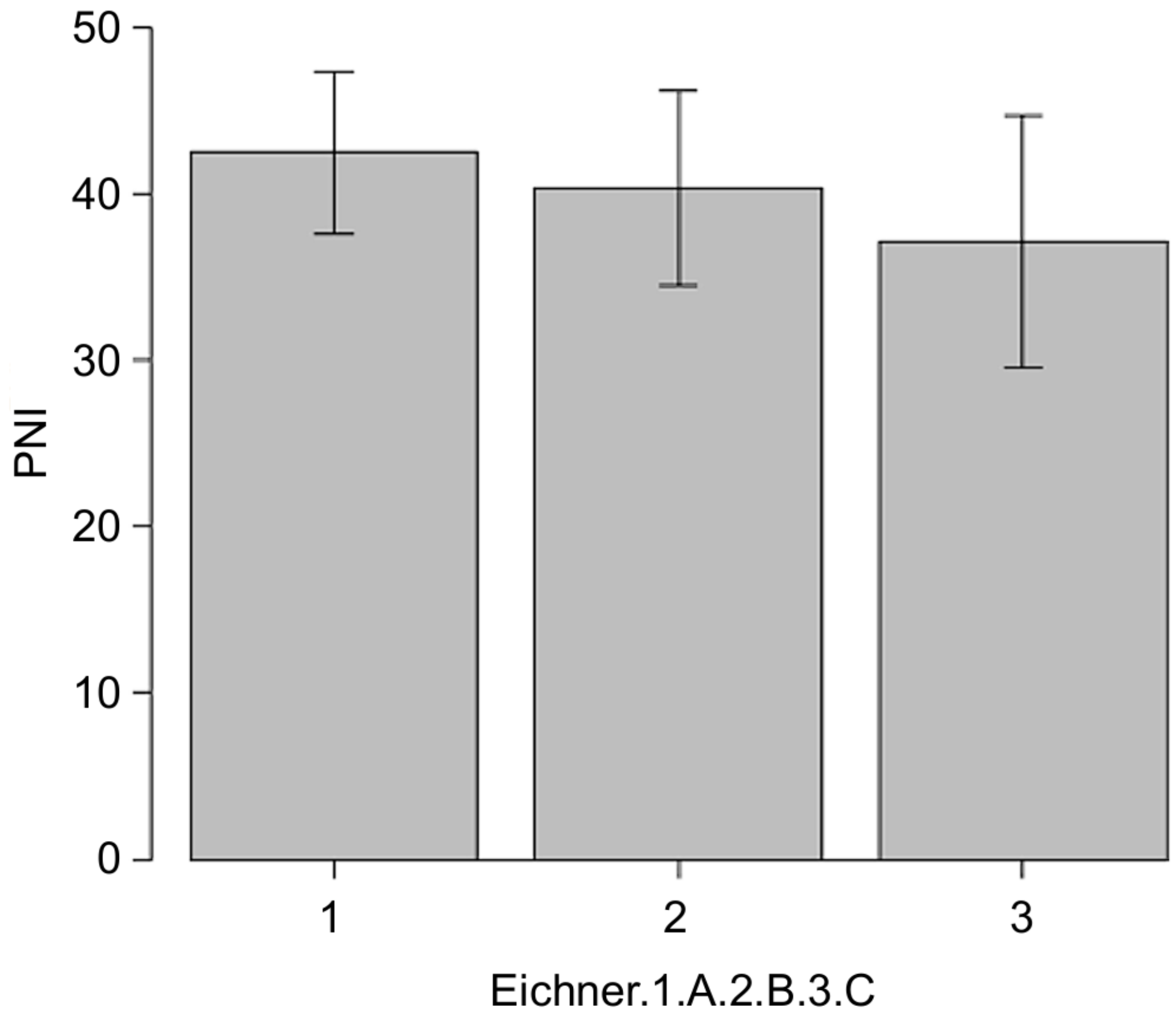

Figure 3

Comparison of PNI levels among the three groups based on Eichner index. PNI: Prognositic nutrition index 


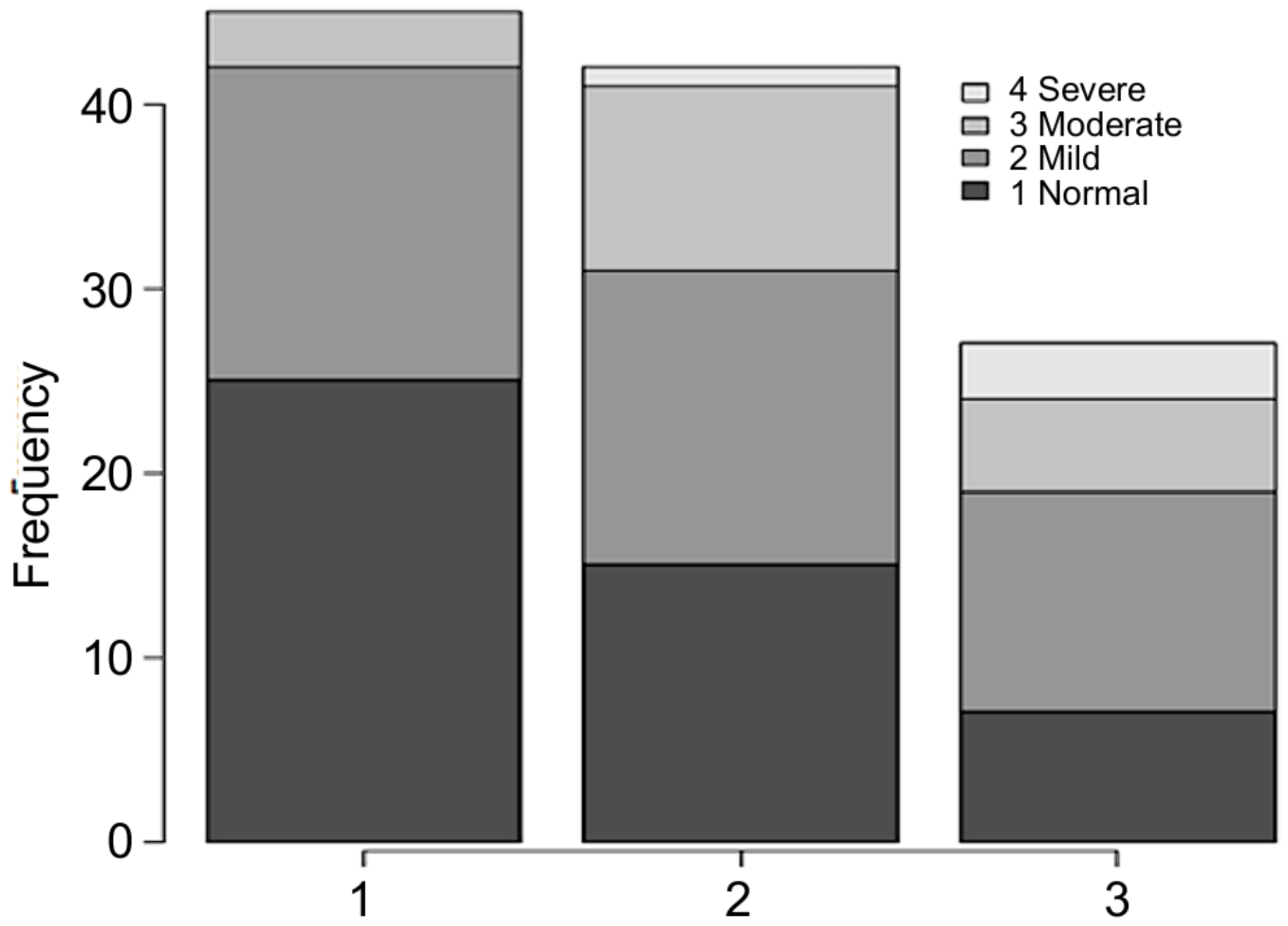

Eichner.1.A.2.B.3.C

Figure 4

Comparison of CONUT levels among the three groups based on Eichner index. CONUT: controlling nutritional status 